\title{
Characterization of adaptive optics point spread function for anisoplanatic imaging. Application to stellar field deconvolution
}

\author{
T. Fusco, J.-M. Conan, L.M. Mugnier, V. Michau, and G. Rousset \\ ONERA, BP. 72, F-92322 Chatillon Cedex, France
}

Received November 10; accepted December 22, 1999

\begin{abstract}
The point spread function (PSF) of an adaptive optics system evolves in the Field Of View (FOV). This variation strongly limits the conventional deconvolution methods for the processing of wide FOV images. A theoretical expression of this PSF variation is derived. This expression is both validated on simulations and experimental data. It is then applied to the a posteriori processing of stellar fields. Using the available prior information about the object (point-like sources), this technique allows the restoration of the star parameters (positions and intensities) with a precision much better than the conventional methods, in a FOV much larger than the isoplanatic field.
\end{abstract}

Key words: instrumentation: adaptive optics techniques: miscellaneous — astrometry — methods: observational

\section{Introduction}

Atmospheric turbulence severely limits the angular resolution of ground based telescopes. Adaptive Optics (AO) (Rousset et al. 1990; Roddier 1999) is a powerful technique to overcome this limitation and to reach the diffraction limit of large telescopes. AO compensates, in real-time, for the random fluctuation of the wavefront induced by the turbulent atmosphere. The turbulent wavefront is measured by a wavefront sensor (WFS) and optically corrected by a deformable mirror. This compensation allows to record high spatial resolution long exposure images. However, even if the object spatial frequencies are preserved up to the diffraction limit of the telescope, they are often severely attenuated since AO correction is only partial. A degraded point spread function (PSF) still blurs the object. It is therefore

Correspondence to:

Tel: (33) 146734040 ; Fax: (33) 146734171.

e-mail: $\{$ NAME\}@onera.fr, URL: http://www.onera.fr/dota necessary to use image processing techniques to improve the quality of the recovered object (Lucy 1994; Thiébaut \& Conan 1995; Christou et al. 1997; Conan et al. 1998b). Nevertheless, all these techniques are based on the assumption that the field of view (FOV) of interest is smaller than the so-called isoplanatic patch (Fried 1982). Wavefronts, coming from angularly separated points, do not cross the same part of the atmosphere and are not identically disturbed. In the visible, the isoplanatic patch is about a few arcseconds (Fried 1982). If the FOV is greater than this field, the AO correction, which is optimal on the optical axis, is degraded as a function of angle (Chassat 1989; Sasiela 1995; Molodij \& Rousset 1997). The residual PSF is no longer space invariant: this fundamentally limits the performance of all the deconvolution techniques.

We present here a simple and analytical expression of this PSF degradation in the FOV and an application to a posteriori processing of wide FOV images.

After a short presentation of the image formation in a wide FOV, a theoretical calculation of the PSF angular dependence is presented in Sect. 2. The results are validated on simulations in Sect. 3 and on experimental data in Sect. 4. In Sect. 5, an example of application is proposed: post processing of an $\mathrm{AO}$ corrected image of a stellar field. A deconvolution algorithm, presented in (Fusco et al. 1999b), is modified to include the theoretical expression of the PSF spatial variation, in order to obtain accurate photometric and astrometric estimations for wide FOV images. This algorithm is then tested on simulated and experimental data.

\section{Theoretical estimation of the PSF angular dependence}

Let $o\left(\boldsymbol{\alpha}^{\prime}\right)$ be the object of interest. For wide FOV, the AO corrected image is given by:

$$
i(\boldsymbol{\alpha})=\int o\left(\boldsymbol{\alpha}^{\prime}\right) h\left(\boldsymbol{\alpha}-\boldsymbol{\alpha}^{\prime}, \boldsymbol{\alpha}^{\prime}\right) \mathrm{d} \boldsymbol{\alpha}^{\prime}+b(\boldsymbol{\alpha})
$$

where $\boldsymbol{\alpha}$ is the angle in the FOV, $h\left(\boldsymbol{\alpha}, \boldsymbol{\alpha}^{\prime}\right)$ the space variant long exposure PSF and $b$ an additive zero mean noise. 
Note that, within the isoplanatic patch, Eq. (1) becomes a convolution between the object and the PSF. In order to well estimate the object $o\left(\boldsymbol{\alpha}^{\prime}\right)$ an accurate knowledge of the PSF $h\left(\boldsymbol{\alpha}, \boldsymbol{\alpha}^{\prime}\right)$ in the whole FOV, is needed.

The computation of the PSF requires the use of the residual phase second order statistics. The long exposure Optical Transfer Function (OTF) (Fourier transform of the PSF) is given by:

$$
\begin{aligned}
\mathrm{OTF}_{\alpha}(\mathbf{f})= & \int \exp \left\{-\frac{1}{2}\left\langle\left[\Phi_{\mathrm{res}, \alpha}(\boldsymbol{\rho})-\Phi_{\mathrm{res}, \alpha}(\boldsymbol{\rho}+\lambda \mathbf{f})\right]^{2}\right\rangle\right\} \\
& \times P(\boldsymbol{\rho}) P(\boldsymbol{\rho}+\lambda \mathbf{f}) \mathrm{d} \boldsymbol{\rho}
\end{aligned}
$$

where $P(\boldsymbol{\rho})$ is the telescope pupil function, $\Phi_{\text {res }, \alpha}(\boldsymbol{\rho})$ the residual phase in the pupil after $\mathrm{AO}$ correction and $\langle$. denotes the expectation over turbulence realizations. The stationarity of the turbulent phase on the telescope pupil is well known. Conan (1994) and Véran (1997) have shown that the AO corrected phase is still quasi-stationary. Then, with this assumption, the OTF expression becomes:

$$
\mathrm{OTF}_{\alpha}(\mathbf{f}) \simeq T_{0}(\mathbf{f}) \exp \left\{-\frac{1}{2} D_{\alpha}(\lambda \mathbf{f})\right\}
$$

where $T_{0}(\mathbf{f})$ is the telescope transfer function without atmospheric turbulence, and with $D_{\alpha}(\lambda \mathbf{f})$ the spatially average residual phase structure function:

$$
\begin{aligned}
& D_{\alpha}(\lambda \mathbf{f})= \\
& \frac{\int\left\langle\left[\Phi_{\mathrm{res}, \alpha}(\boldsymbol{\rho})-\Phi_{\mathrm{res}, \alpha}(\boldsymbol{\rho}+\lambda \mathbf{f})\right]^{2}\right\rangle P(\boldsymbol{\rho}) P(\boldsymbol{\rho}+\lambda \mathbf{f}) \mathrm{d} \boldsymbol{\rho}}{\int P(\boldsymbol{\rho}) P(\boldsymbol{\rho}+\lambda \mathbf{f}) \mathrm{d} \boldsymbol{\rho}}
\end{aligned}
$$

in a direction $\alpha$ and for a wavelength $\lambda$. Introducing the on-axis structure function Eq. (4) can be re-written as

$$
D_{\alpha}(\lambda \mathbf{f})=D_{0}(\lambda \mathbf{f})+D_{\text {ani }}(\lambda \mathbf{f}, \alpha) .
$$

where $D_{\text {ani }}$ is defined as follows:

$$
\begin{aligned}
& D_{\mathrm{ani}}(\lambda \mathbf{f}, \alpha)= \\
& \left(\int \left\langle\left[\Phi_{\mathrm{res}, \alpha}(\boldsymbol{\rho})-\Phi_{\mathrm{res}, \alpha}(\boldsymbol{\rho}+\lambda \mathbf{f})\right]^{2}\right.\right. \\
& \left.\quad-\left[\Phi_{\mathrm{res}, 0}(\boldsymbol{\rho})-\Phi_{\mathrm{res}, 0}(\boldsymbol{\rho}+\lambda \mathbf{f})\right]^{2}\right\rangle \\
& \quad \times P(\boldsymbol{\rho}) P(\boldsymbol{\rho}+\lambda \mathbf{f}) \mathrm{d} \boldsymbol{\rho}) /\left(\int P(\boldsymbol{\rho}) P(\boldsymbol{\rho}+\lambda \mathbf{f}) \mathrm{d} \boldsymbol{\rho}\right)
\end{aligned}
$$

$\mathrm{OTF}_{\alpha}$ is the product of $\mathrm{OTF}_{0}$, the OTF on the optical axis, with a term which can be called "anisoplanatic transfer function" (ATF) and reads:

$$
\mathrm{OTF}_{\alpha}(\mathbf{f})=\mathrm{OTF}_{0}(\mathbf{f}) \operatorname{ATF}(\mathbf{f}, \alpha)
$$

with

$$
\operatorname{ATF}(\mathbf{f}, \alpha)=\exp \left\{-\frac{1}{2} D_{\text {ani }}(\lambda \mathbf{f}, \alpha)\right\}
$$

Note that, on-axis means here: on the wavefront sensor optical axis. $\mathrm{OTF}_{0}(\mathbf{f})$ can be estimated using the method proposed by Véran (1997) which is based on real-time data statistics accumulated by the AO control system during the observation. To obtain a theoretical expression of the $\mathrm{ATF}, D_{\text {ani }}(\lambda \mathbf{f}, \alpha)$ must be computed.

Let us use the modal decomposition of the turbulent phase onto the Zernike polynomial basis:

$$
\Phi(\boldsymbol{\rho})=\sum_{i=2}^{\infty} a_{i} Z_{i}(\boldsymbol{\rho}) .
$$

The properties of turbulence in this particular basis are described by Noll (1976). In a first approximation, the AO correction can be seen as a high-pass filter which provides a full correction of all the Zernike polynomials up to a given number. With this assumption and for an AO correction up to the polynomial number $i_{0}$, we have:

$$
\begin{aligned}
& \Phi_{\mathrm{res}, 0}(\boldsymbol{\rho})=\sum_{i=i_{0}+1}^{\infty} a_{i}(0) Z_{i}(\boldsymbol{\rho}) \text { and } \\
& \Phi_{\mathrm{res}, \alpha}(\boldsymbol{\rho})=\sum_{i=2}^{i_{0}}\left(a_{i}(\alpha)-a_{i}(0)\right) Z_{i}(\boldsymbol{\rho})+\sum_{i=i_{0}+1}^{\infty} a_{i}(\alpha) Z_{i}(\boldsymbol{\rho})
\end{aligned}
$$

where $a_{i}(0)$ and $a_{i}(\alpha)$ are the Zernike coefficients of the phase expansion on the optical axis and for a direction $\alpha$ respectively. Using this expansion, a theoretical expression of $D_{\text {ani }}(\lambda \mathbf{f}, \alpha)$ can be obtained:

$$
D_{\text {ani }}(\lambda \mathbf{f}, \alpha)=\sum_{i=1}^{i_{0}} \sum_{j=1}^{\infty} 2\left[C_{i, j}(0)-C_{i, j}(\alpha)\right] U_{i j}(\lambda \mathbf{f})
$$

where $C_{i, j}(\alpha)$ are the angular correlation of the Zernike coefficients $a_{i}$ and $a_{j}$,

$$
C_{i, j}\left(\alpha_{2}-\alpha_{1}\right)=C_{i, j}\left(\alpha_{1}-\alpha_{2}\right)=\left\langle a_{i}\left(\alpha_{1}\right) a_{j}\left(\alpha_{2}\right)\right\rangle
$$

and $U_{i j}$ are functions defined as:

$U_{i j}(\lambda \mathbf{f})=$

$\frac{\int\left[Z_{i}(\boldsymbol{\rho})-Z_{i}(\boldsymbol{\rho}+\lambda \mathbf{f})\right]\left[Z_{j}(\boldsymbol{\rho})-Z_{j}(\boldsymbol{\rho}+\lambda \mathbf{f})\right] P(\boldsymbol{\rho}) P(\boldsymbol{\rho}+\lambda \mathbf{f}) \mathrm{d} \boldsymbol{\rho}}{\int P(\boldsymbol{\rho}) P(\boldsymbol{\rho}+\lambda \mathbf{f}) \mathrm{d} \boldsymbol{\rho}}$.

The angular correlations $C_{i, j}(\alpha)$ can be theoretically computed (Chassat 1989) assuming that the $C_{n}^{2}$ profile is known. Note that a crude estimation of the $C_{n}^{2}$ is enough because of the weak dependency of the angular correlation of the phase with the atmospheric profile (Chassat 1989; Molodij \& Rousset 1997). Nevertheless, a good estimation of the $C_{n}^{2}$ profile can be obtained by a SCIDAR measurement (Fuchs et al. 1998), for example.

Equation (12) is a generalization of the expression given by Voitsekhovich \& Bara (1999) who consider the case of a perfect correction $\left(\Phi_{\mathrm{res}, 0}(\boldsymbol{\rho})=0\right)$ on the optical axis. Note that, because of the difference between the correlations $C_{2,2}(\alpha)$ and $C_{3,3}(\alpha)$ (Chassat 1989), Eq. (12) gives an anisotropic ATF which leads to an elongated PSF (Voitsekhovich \& Bara 1999; Close et al. 1998).

Using Eqs. (7), (8) and (12), the OTF in the whole field of view can be theoretically computed, assuming an infinite exposure time. 


\section{Comparison between theory and simulation}

\subsection{Turbulence simulation method}

In order to validate the Eqs. (5) and (12) derived in Sect. 2, wide FOV AO images are simulated. We consider a three layer $C_{n}^{2}$ profile: one on the pupil, the second at one $\mathrm{km}$ and the third at ten $\mathrm{km}$ with respectively 20,60 and $20 \%$ of the turbulence. Using the so-called near-field approximation (Roddier 1981), the scintillation effects are neglected and the phase on the telescope pupil for a given direction $\boldsymbol{\alpha}$ is simply the sum of the corresponding part of each phase screen in each turbulent layer:

$$
\Phi_{\boldsymbol{\alpha}}(\boldsymbol{\rho})=\sum_{j=1}^{3} \varphi_{j}\left(\boldsymbol{\rho}+h_{j} \boldsymbol{\alpha}\right)
$$

where $\varphi_{j}$ and $h_{j}$ are respectively the phase screen and the height of the $j^{\text {th }}$ layer. Each phase screen is simulated by N. Roddier's method (Roddier 1990) using the 861 first Zernike polynomials (radial order up to 40). The size of these phase screens corresponds to a 20 arcsecs FOV radius and a telescope diameter of $4 \mathrm{~m}$. The overall $D / r_{0}$ is 10 .

\subsection{Long exposure PSF simulation}

Let us consider an adaptive optics system which can perfectly correct the $i_{0}$ first Zernike polynomials, in our case, $i_{0}=21$. We have simulated a PSF at every arcsecond in the FOV (20 arcsec). Each PSF is the sum of 1000 time-decorrelated short exposures which are deduced from $\Phi_{\text {res }, \alpha}(\boldsymbol{\rho})$. If we consider that the speckle lifetime is about $10 \mathrm{~ms}$, the simulation are roughly equivalent to a $10 \mathrm{~s}$ long exposure. Figure 1 shows the PSF evolution as a function of angle. Note that the PSF does not vary significantly within 3 arcsec, which roughly corresponds to the isoplanatic angle given by Roddier (1981):

$$
\theta_{0}=0.314 \frac{r_{0}}{\bar{h}}=1.64^{\prime \prime}
$$

with $\bar{h}$ given by (Fried 1982)

$$
\bar{h}=\left[\frac{\int_{0}^{\infty} h^{\frac{5}{3}} C_{n}^{2}(h) \mathrm{d} h}{\int_{0}^{\infty} C_{n}^{2}(h) \mathrm{d} h}\right]^{\frac{3}{5}}=3953 m .
$$

In this field, the PSF variations are only due to the turbulence noise (finite number of short exposures in the PSF calculation). Outside the isoplanatic field, the PSF degradation is important, the Strehl Ratio goes from $43 \%$ at the center of the field to less than $10 \%$ at the border of the field. Of course, a deconvolution scheme, assuming that the PSF is constant in the whole field of view, would lead to a poor restoration. Note that the non-circularity of the off-axis PSF's appears in Fig. 1.

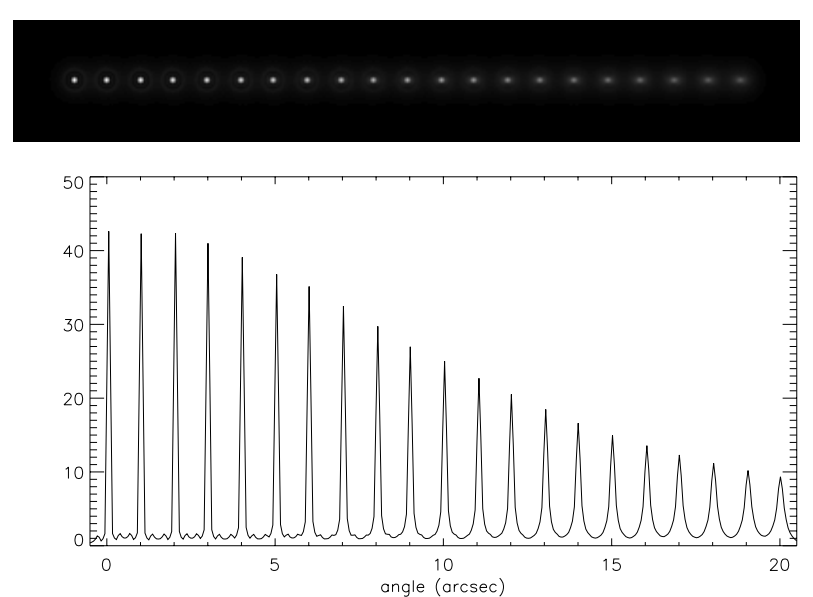

Fig. 1. PSF in the FOV versus angle. On the top: $2 \mathrm{D}$ images of the simulated PSF's for each arcsecond. On the bottom: $\mathrm{X}$-cut of the images. The PSF's normalized by the diffraction (in percent)

\subsection{Results}

For each angle, we compare the theoretical OTF (see Eqs. 7 and 8) to the simulated one. A very good estimation for each OTF is obtained, even in the case of large values of $\alpha$ (see Fig. 2).

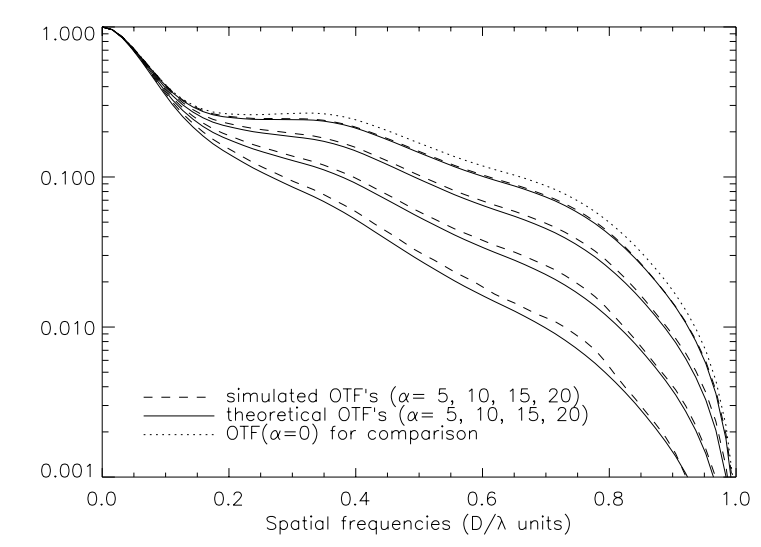

Fig. 2. $Y$-cut of the simulated OTF (dashed line) and theoretical OTF given by Eq. (7) (solid line) for $\alpha=5,10,15$ and $20^{\prime \prime}$. The OTF for $\alpha=0$ is shown for comparison

Because we only consider a $Y$-cut of the OTF's in Fig. 2, the anisotropic (elongation) effect is not visible. In Fig. 3 we only consider one angle $\left(\alpha=20^{\prime \prime}\right)$ but we plot two cuts ( $X$ and $Y$ axis) of the simulated and theoretical OTF. In that case, the anisotropic effect and its good restitution by the analytical ATF is shown.

A small under-estimation of the OTF can be seen in each case with the analytical method. The difference between the simulated and theoretical OTF's is due to the phase non-stationarity error. In Sect. 2 we have assumed 


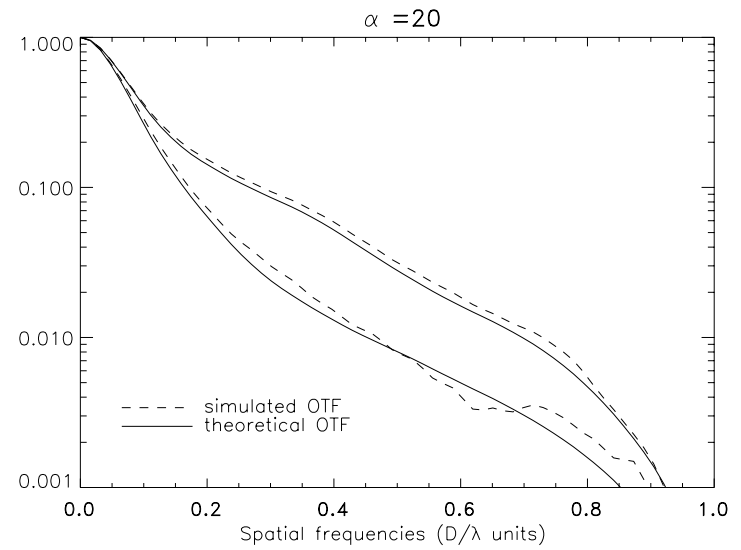

Fig. 3. $Y$-cut and $X$-cut of the simulated OTF (dashed line) and the theoretical OTF (solid line) for $\alpha=20^{\prime \prime}$

the phase stationarity on the telescope pupil. This assumption corresponds to approximate the mean of the exponential of function by the exponential of the mean of the function. Even if this approximation is quite good (Véran 1997), it leads, nevertheless, to a small under estimation of the theoretical OTF. This error gives the fundamental limitation of the method, but in experimental data processing, other error sources may also degrade the results, such as:

- atmospheric parameter estimation errors on: $r_{0}, L_{0}, C_{n}^{2}$ profile;

- AO full correction of the first Zernike modes which is only an approximation of a real system;

- turbulent noise: finite exposure time which leads to a residual speckle pattern in the long exposure image.

But we show in the next section, that in a real case, all these errors do not significantly influence the ATF estimation.

\section{Experimental results}

\subsection{Observations}

The theoretical estimation of OTF angular variation is compared with experimental data recorded in September 1997 with the ONERA adaptive optics bench installed on the $1.52 \mathrm{~m}$ telescope at the Observatoire de Haute Provence (Conan et al. 1998a). The Adaptive Optics Bench (BOA) is a 88 actuator system, with a ShackHartmann wave-front sensor (64 sub-apertures). Two images of two separated binary stars ( $\xi$ Cephee and $\Theta$ Orionis) were acquired at $850 \mathrm{~nm}$, with a 10 second integration time, on the 29 September 1997 at $00 \mathrm{~h} 02$ and $04 \mathrm{~h} 27$ (UT). The visual magnitude of the two components are: 4.6, 6.5 for $\xi$ Chepee and 5.1, 6.7 for $\Theta$ Orionis. The separation of these binary stars is large enough (about 8 and 13 arcsec) to obtain two PSF's with no overlap (see
Fig. 4). In each case, the brightest star is used for the wavefront sensing. During the same night at $21 \mathrm{~h} 21$ (UT), a $C_{n}^{2}$ profile measurement was obtained by M. Azouit from Université de Nice (France) (see Fig. 5) using balloon probes (Vernin \& Munoz-Tunón 1992). For the two images the observing conditions are summarized in Table 1. $r_{0}$ has been estimated using open-loop Shack Hartmann data recorded shortly before the corrected images and $\theta_{0}$ has been computed using $r_{0}$, measured $C_{n}^{2}$ profile and Eq. (16).

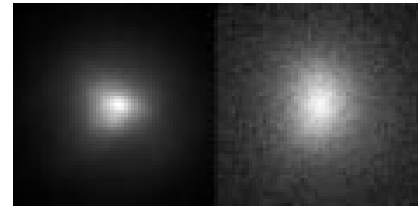

a

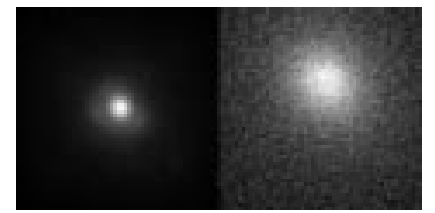

$\mathrm{b}$
Fig. 4. Images of the two components of: a) $\xi$ Cephee (separation $\left.\simeq 8^{\prime \prime}\right)$ b) $\Theta$ Orionis (separation $\left.\simeq 13^{\prime \prime}\right)$. 1 pixel $=$ 0.027 arcsec, image size $=64$ pixels

Table 1. Observing conditions for $\xi$ Cephee and $\Theta$ Orionis. In the two cases, the sampling frequency is equal to $264 \mathrm{~Hz} . \theta_{0}$ is the isoplanatic angle defined with Eq. (16), $L_{0}$ is the outer scale, and SR the Strehl ratio given in percent

\begin{tabular}{|c||c|c|c|}
\hline & $\begin{array}{c}r_{0}(\text { in } \mathrm{m}) \\
(@ 500 \mathrm{~nm})\end{array}$ & $L_{0}($ in $\mathrm{m})$ & $\begin{array}{c}D / r_{0} \\
(@ 850 \mathrm{~nm})\end{array}$ \\
\hline$\xi$ Cephee & 0.055 & 4 & 14.6 \\
\hline$\Theta$ Orionis & 0.06 & 4 & 13.4 \\
\hline \hline & $\begin{array}{c}\theta_{0}(\operatorname{arcsec}) \\
(@ 850 \mathrm{~nm})\end{array}$ & $\begin{array}{c}\text { SR } \\
\text { PSF } 1\end{array}$ & $\begin{array}{c}\text { SR } \\
\text { PSF } 2\end{array}$ \\
\hline$\xi$ Cephee & 1.53 & $4 \%$ & $2 \%$ \\
\hline$\Theta$ Orionis & 1.66 & $8 \%$ & $3 \%$ \\
\hline
\end{tabular}

\subsection{ATF computation}

We have approximated this $C_{n}^{2}$ profile by 3 "equivalent layers" (EL) (Fusco et al. 1999a). The true $C_{n}^{2}$ profile is divided in 3 slabs ( 0 to $1 \mathrm{~km}, 1$ to $10 \mathrm{~km}$ and 10 to $27 \mathrm{~km}$ ), for each slab an EL is located at an equivalent height defined as the weighted mean height of the slab: $h_{\mathrm{eq}}=\left(\int_{h_{\min }}^{h_{\max }} C_{n}^{2}(h) h \mathrm{~d} h\right) /\left(\int_{h_{\min }}^{h_{\max }} C_{n}^{2}(h) \mathrm{d} h\right)$ and with an associated strength given by the total strength of the slab $\left(\int_{h_{\min }}^{h_{\max }} C_{n}^{2}(h) \mathrm{d} h\right)$. All the values are summarized in Table 2 .

Because of the weak dependency of the angular decorrelation of the phase with the atmospheric profile, this $C_{n}^{2}$ approximation is enough to obtain a good estimate on 
Table 2. Results of the equivalent layer calculations

\begin{tabular}{|c||c|c|c|c|}
\hline & $h_{\min }$ & $h_{\max }$ & $\begin{array}{c}\text { equivalent } \\
\text { height } \\
\text { (in } \mathrm{km} \text { ) }\end{array}$ & $\begin{array}{c}\text { equivalent } \\
\text { strength } \\
\text { (in \%) }\end{array}$ \\
\hline layer 1 & 0 & 1 & 7.7 & 56.5 \\
\hline layer 2 & 1 & 10 & 3.3 & 33.5 \\
\hline layer 3 & 10 & 27 & 14.1 & 10 \\
\hline
\end{tabular}

the phase angular decorrelation. We checked that an increase of the layer number (three to one hundred) do not significantly change the results.

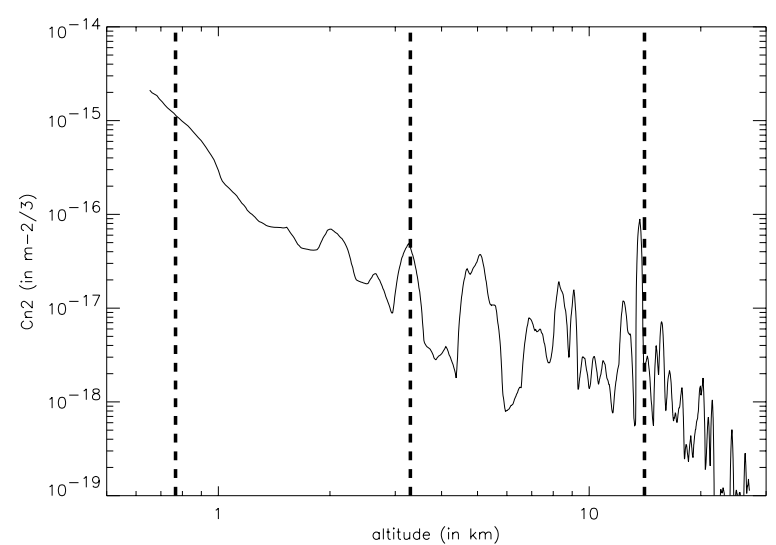

Fig. 5. $C_{n}^{2}$ profile measurements obtained with balloon probes by M. Azouit [solid line] and position of the 3 equivalent layers [dashed line]

In order to estimate the OTF, the AO system is assumed to provide a perfect correction of a given number of Zernike polynomials. This number $N$ is defined so that the $N$ first modes have a wavefront reconstruction $S N R$ higher than 1. For this purpose, we use the theoretical behavior of the turbulent and of the noise variance (Rigaut \& Gendron 1992). These variances are scaled using the measured values of $r_{0}$ and $L_{0}$ for the Zernike turbulent variance and by the propagation of the photon and detector noise in the wavefront sensor through the reconstruction matrix for the Zernike noise variance (Rousset 1999).

With the observing conditions, the correction in terms of Zernike polynomials is efficient $(S N R \geq 1)$ about up to the $10^{\text {th }}$ Zernike for $\xi$ Cephee and the $15^{\text {th }}$ Zernike for $\Theta$ Orionis.

Now, we compute the OTF for each binary star component (Eq. 7): the theoretical ATF is given by Eqs. (12) and (8) using:

- the 3 EL's profile for the computation of the angular correlation of the Zernike;

- the first $10^{\text {th }}$ (resp. $15^{\text {th }}$ ) Zernike coefficients;

- the known separation between the two stars.

The on-axis OTF is given by the first component of each binary star (brightest star).
The comparison between the measured OTF and the computed OTF is shown in Fig. 6.
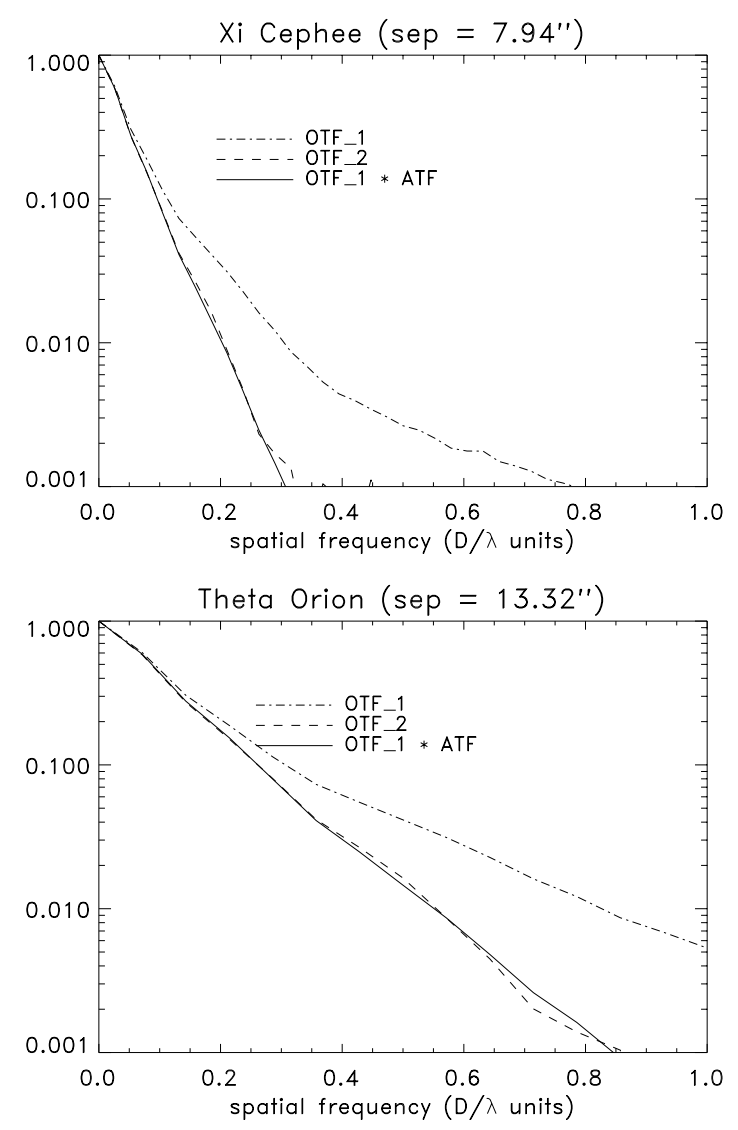

Fig. 6. Comparison between the measured [dashed line] and the computed [solid line] off-axis OTF. The measured OTF for $\alpha=0$ (brightest star) [dotted-dashed line] is given for comparison: (top) $\xi$ Cephee and (bottom) $\Theta$ Orionis. The circular average of each OTF is plotted

In the two cases, a good accordance between measured and computed OTF is found although a large number of assumptions has been made to compute the ATF:

- $C_{n}^{2}$ constant during the whole night (3h $40 \mathrm{mn}$ and $8 \mathrm{~h}$ between the $C_{n}^{2}$ measurement and the two observations). Even if we know that the sensitivity to this profile is low; - $r_{0}$ and $L_{0}$ estimation from the WFS noisy data assumed to be perfect;

- efficient correction of respectively the first 10 and 15 Zernike coefficients, which is a crude approximation of the AO system.

These results are very encouraging and allow to be optimistic for large FOV image post-processing.

Now, let us use the theoretical model of the PSF degradation for the post-processing of AO wide FOV images of stellar fields. 


\section{Application to stellar field post-processing}

One of the first applications of wide FOV imaging in astronomy is the observation of large stellar fields. In this case, we have a strong a priori information on the object: it is a sum of Dirac functions (Gunsay \& Jeffs 1995; Fusco et al. 1999b):

$$
o(\boldsymbol{\alpha})=\sum_{k=1}^{n} \gamma_{k} \delta\left(\boldsymbol{\alpha}-\boldsymbol{\alpha}_{k}\right)
$$

where $\boldsymbol{\alpha}_{k}$ and $\gamma_{k}$ are the position vector and the intensity of the $k^{\text {th }}$ star respectively. They are the object parameters we are seeking. We consider that $n$, the number of stars, is a known parameter. Note that, using appropriate prior statistics (Bernouilli Gaussian or Poisson-Gaussian for example), it is possible to generalize the method and to incorporate $n$ as an unknown of the problem.

\subsection{The deconvolution method}

To solve this inverse problem, i.e., estimate the unknowns $\left(\boldsymbol{\alpha}_{k}, \gamma_{k}\right)$, we use the approach presented in (Fusco et al. 1999b) with a isoplanatic PSF, but modified to include the anisoplanatic effects. Note that this appraoch can be seen as a "PSF fitting" in the Fourier domain.

The criterion to be minimized with respect to $\alpha_{k}$ and $\gamma_{k}$ can be written in the Fourier domain as:

$$
\begin{aligned}
& J\left(\gamma_{k}, \boldsymbol{\alpha}_{k}\right)= \\
& \left\|\sum_{k=1}^{n} \gamma_{k} \exp \left(-2 j \pi \mathbf{f} . \boldsymbol{\alpha}_{k}\right) \times \mathrm{OTF}_{\boldsymbol{\alpha}_{k}}(\mathbf{f})-\tilde{i}(\mathbf{f})\right\|^{2}
\end{aligned}
$$

where $\mathrm{OTF}_{\boldsymbol{\alpha}_{k}}(\mathbf{f})$ is the OTF for the $k^{\text {th }}$ star and $\mathbf{f}$ the spatial frequency and . denotes a Fourier transform. $\mathrm{OTF}_{\boldsymbol{\alpha}_{k}}(\mathbf{f})$ is slowly variable with $\alpha_{k}$. Then a crude estimation of $\alpha_{k}$ using a first deconvolution with a constant PSF in the whole field of view allows the calculation of the associated ATF's. In a first approximation, these ATF estimates are assumed to be the true ones and are not adjusted in the deconvolution process. The criterion to be minimized is then:

$$
\begin{gathered}
J\left(\gamma_{k}, \boldsymbol{\alpha}_{k}\right)=\| \sum_{k=1}^{n} \gamma_{k} \exp \left(-2 j \pi \mathbf{f} . \boldsymbol{\alpha}_{k}\right) \times \mathrm{OTF}_{0}(\mathbf{f}) \times \\
\operatorname{ATF}_{\alpha_{k}}(\mathbf{f})-\tilde{i}(\mathbf{f}) \|^{2} .
\end{gathered}
$$

Note that a more complete, but more complex approach should consider $\mathrm{ATF}_{\alpha_{k}}(\mathbf{f})$ as unknow of the problem.

This minimization is done using a conjugate gradient method. The object reparametrization allows an accurate precision on the parameters (sub-pixel precision on the star positions). Nevertheless, the criterion is not convex and a good first initialization for the star positions is suitable to avoid problems related to local minima.
This initialization is made using a pixel by pixel deconvolution (Wiener filter or Lucy Richardson Algorithm for example).

Let us consider an object which is a field of 21 stars with the same magnitude. The separation between each stars is one arcsecond (see Fig. 1). In order to focus on the limitation induced by the anisoplanatic problem and the gain brought by the ATF estimation, we first consider a noise free image, but the noise influence will be discussed later in the paper.

\subsection{Noise free image}

We compare, on a noise free image (see Fig. 1), the gain brought by the ATF introduction in the image processing. ATF's for each star are computed using an estimation of $\alpha_{k}$ given by the first pixel by pixel deconvolution (Wiener filter). $\mathrm{OTF}_{0}$ is simply given by the fourier transform of the on-axis PSF. We plot in Fig. 7 the error, given in percent, on the stars magnitude estimate using "isoplanatic" deconvolution process (deconvolution using criterion defined in Eq. (18) but assuming that the PSF is constant in the whole FOV) and our modified approach using the ATF estimation for each star position Eq. (19). Figure 7 shows the gain brought by the use of the ATF in the a posteriori processing. The error, which increases as a function of angle, in the "isoplanatic" deconvolution case, up to $34 \%$ for $\alpha=20^{\prime \prime}$, is close to be constant and only about $1 \%$ when we use the ATF estimation. We believe that this residual error is due to the non-stationarity assumption made in the ATF estimation (see Sect. 3.3).

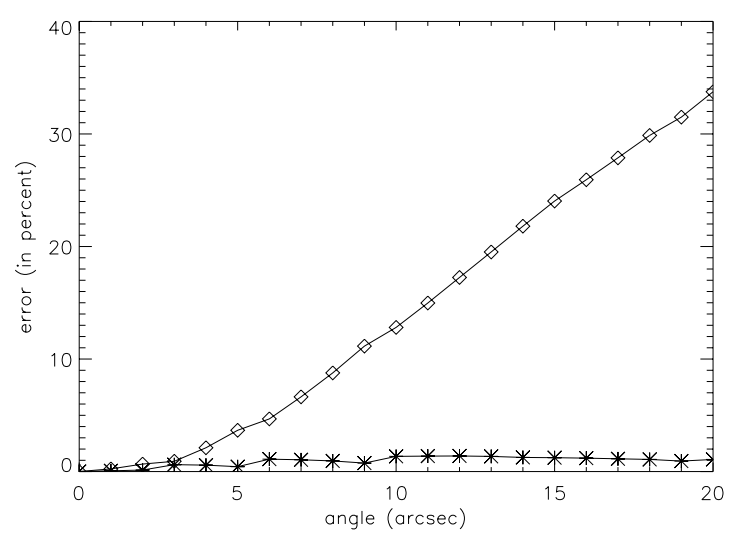

Fig. 7. Error in percent on the magnitude estimation for each star in the case of a noise free image. $[\diamond]$ estimation using the same PSF $(\alpha=0)$ for the whole FOV, $[*]$ estimation using the ATF associated to each star 


\subsection{Influence of the photon noise}

Of course, in a realistic case, the image is not only degraded by the turbulence effect but also affected by photon and detector noise. Let us assume that in the case of AO long exposures, the dominant noise is the photon noise. It leads to an error on the parameter estimation.

In the case of a "isoplanatic" deconvolution (PSF constant in the whole FOV), for a given flux (that is for a given noise level), a limit angle can be defined below which the anisoplanatic error is lower than the noise error. The lower the flux, the greater the limit angle (see Fig. 8). For example, in the case of $10^{3}$ photons the anisoplanatic error becomes greater than the noise error for $\alpha \geq 7^{\prime \prime}$. This limit angle can be seen as a definition of an isoplanatic angle for our deconvolution method.

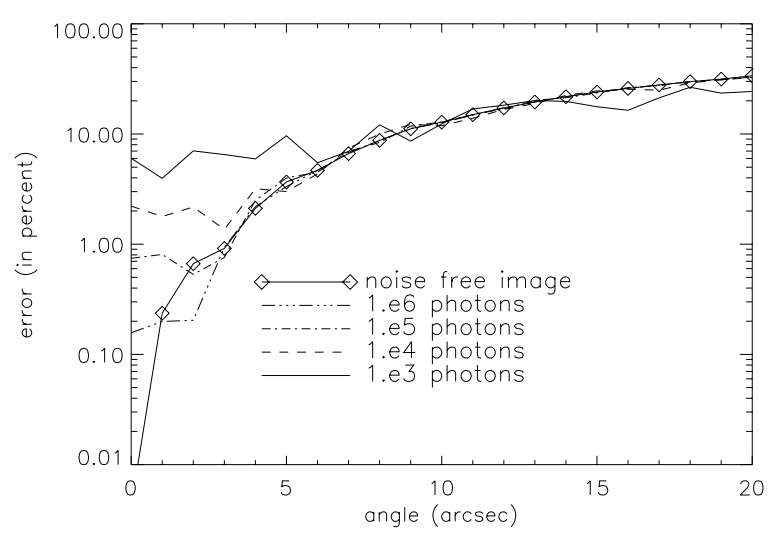

Fig. 8. Error in percent on the magnitude estimation using the same OTF $(\alpha=0)$ in the whole FOV, in the case of a noise free image, and in the case of photon noise with $10^{6}, 10^{5}, 10^{4}$ and $10^{3}$ photons in the whole image respectively. The error is only averaged on 10 noise outcomes for each flux case

Now, let us use the ATF correction of the OTF in the post-processing. In that case, the noise error is dominant for the whole FOV, as soon as it is of the order or greater than $1 \%$ which corresponds to $10^{5}$ photons in our case (see Fig. 9). Even if the ATF under-estimation due to the phase non-stationarity error is the dominant noise for a flux greater than $10^{5}$ photons, the gain brought by the use of the ATF is still very important with respect to a "isoplanatic" deconvolution (see Figs. 8 and 9). And, at low flux, the error in the FOV is only limited by the photon noise.

\subsection{Experimental results}

Let us now apply the wide FOV deconvolution method (Eq. 19) on the two experimental images of $\xi$ Cephee and $\Theta$ Orionis presented in Sect. 4. In these two cases, because

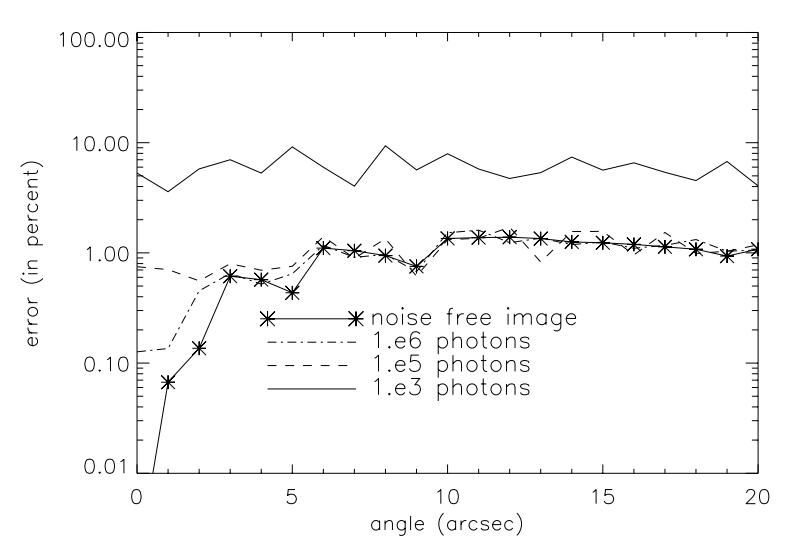

Fig. 9. Error in percent on the magnitude estimation using the ATF correction of the OTF in the post processing in the case of a noise free image, and in the case of photon noise with $10^{6}$, $10^{5}$ and $10^{3}$ photons in the whole image respectively. The error is only averaged on 10 noise outcomes for each flux case

of the large angular separation between the two components, an accurate estimation of the magnitude difference and of the separation can be obtained using the sole images and computing a center of gravity and an integral of the flux for each image. Since we have a good estimation of the parameters of interest by this aperture photometry method, it is interesting to validate our deconvolution approach on this data and to evaluate the gain brought by the ATF estimation.

The results of the estimation of the separations and the magnitude differences are summarized in Table 3 for each processing case:

- a crude aperture photometry for the magnitude estimation plus a center of gravity estimation for the seperation estimation;

- a deconvolution with a sole PSF (on axis PSF, that is the brighter star image) for the whole image ("isoplanatic" deconvolution);

- and the use of the ATF model in the deconvolution process.

These results clearly show the gain brought by the ATF correction of the OTF on the estimation of star parameters: mainly on the magnitude estimation. The "isoplanatic" deconvolution process, which is not efficient in the case of large FOV, because of the PSF variation, is strongly improved by the introduction of the theoretical degradation of this PSF as a function of angle.

\section{Conclusion}

Anisoplanatism is one of the most important limitation of large FOV high resolution imaging. We present a simple and analytical expression to model the OTF variation as a function of field angle. The OTF for an angle $\alpha$ is the 
Table 3. Angular separation and magnitude difference estimation for $\xi$ Cephee and $\Theta$ Orionis using a center of gravity calculation, a "isoplanatic" deconvolution (a sole PSF for the whole image) and a deconvolution with the anisoplanatic OTF

\begin{tabular}{|c||c|c|c|}
\hline$\xi$ Cephee & $\begin{array}{c}\text { Center of } \\
\text { gravity }\end{array}$ & $\begin{array}{c}\text { "Isoplanatic" } \\
\text { deconvolution }\end{array}$ & $\begin{array}{c}\text { ATF estimation } \\
\text { deconvolution }\end{array}$ \\
\hline $\begin{array}{c}\text { Angular } \\
\text { separation }\end{array}$ & $7.95^{\prime \prime}$ & $7.94^{\prime \prime}$ & $7.95^{\prime \prime}$ \\
\hline $\begin{array}{c}\text { Magnitude } \\
\text { difference }\end{array}$ & 1.89 & 2.01 & 1.87 \\
\hline \hline$\Theta$ Orionis & $\begin{array}{c}\text { Center of } \\
\text { gravity }\end{array}$ & $\begin{array}{c}\text { "Isoplanatic" } \\
\text { deconvolution }\end{array}$ & $\begin{array}{c}\text { ATF estimation } \\
\text { deconvolution }\end{array}$ \\
\hline $\begin{array}{c}\text { Angular } \\
\text { separation }\end{array}$ & 13.30 & 13.45 & 13.41 \\
\hline $\begin{array}{c}\text { Magnitude } \\
\text { difference }\end{array}$ & 1.48 & 1.96 & 1.50 \\
\hline
\end{tabular}

product of the on-axis OTF by an anisoplanatic transfer function (ATF) which only depends on the angular decorrelation of the phase. The incorporation of this ATF in a post processing of large FOV images corrected by $\mathrm{AO}$ leads to good accuracy on the object parameter estimation. This method has been validated both on simulated and experimental data. In the case of a stellar field, the relative error on the magnitude estimation can be divided by more than an order of magnitude for large angles.

Nevertheless, the OTF estimation is never perfect and a residual error still limits the post-processing performance $($ error $\simeq 1 \%$ ). This residual error is due both to the non-stationarity of the AO compensated phase in the pupil and to the turbulence noise (finite exposure time). Furthermore, the $C_{n}^{2}$ profile must be known to compute the ATF, even if there is a weak dependency of the results with this profile. A SCIDAR (Fuchs et al. 1998) on the astronomic site may provide such an information, but this $C_{n}^{2}$ knowledge may be difficult to obtain in all cases. Another solution to these problems could be to use an approximated parametric model of the ATF in order to perform a joint estimation of the object and of the ATF parameters.

Acknowledgements. This work was supported by contracts from Service Technique des Technologies Communes, Ministère de la Défense, France. The authors wish to thank J.-P. Véran, F. Charbonnier and A. Blanc for fruitful discussions. Many thanks also to M. Azouit for the $C_{n}^{2}$ data acquisition and to P.-Y. Madec, D. Rabaud and B. Fleury who took part to the AO observing run.

\section{References}

Chassat F., 1989, J. Opt. (Paris) 20(1), 13

Christou J.C., Bonaccini D., Ageorges N., 1997, in Tyson R.K. and Fugate R.Q. (eds.), Adaptive Optics and Applications, Vol. 3126, SPIE, Soc. Photo-Opt. Instrum. Eng., Washington, pp. 68-80

Close L.M., Roddier F., Roddier C., Graves J.E., Northcott M., Potter D., 1998, SPIE, Soc. Photo-Opt. Instrum. Eng., Kona, Hawaii, pp. 406-415

Conan J.-M., 1994, Ph.D. Thesis, Université Paris XI Orsay

Conan J.-M., Fusco T., Mugnier L., Kersalé E., Michau V., 1998a, in Astronomy with adaptive optics: present results and future programs, ESO/OSA, Sonthofen

Conan J.-M., Mugnier L., Fusco T., Michau V., Rousset G., 1998b, Appl. Opt. 37(21), 4614

Fried D.L., 1982, J. Opt. Soc. Am. 72(1), 52

Fuchs A., Tallon M., Vernin J., 1998, PASP 110(86)

Fusco T., Conan J.-M., Michau V., Mugnier L., Rousset G., 1999a, Opt. Lett. 24(21)

Fusco T., Véran J.-P., Conan J.-M., Mugnier L., 1999b, A\&AS 134, 1

Gunsay M., Jeffs D., 1995, in IEEE Trans. Image Proc., Vol. 4, IEEE, pp. 1602-1612

Lucy L., 1994, in Hanish R. and White R. (eds.), The Restoration of HST Image and Spectra II, pp. 79-85

Molodij G., Rousset G., 1997, J. Opt. Soc. Am. A 14(8), 1949

Noll R.J., 1976, J. Opt. Soc. Am. 66(3), 207

Rigaut F., Gendron E., 1992, A\&A 261, 677

Roddier F., 1981, in Wolf E. (ed.), Progress in Optics, Vol. XIX, North Holland, Amsterdam, pp. 281-376

Roddier F. (ed.), 1999, Adaptive Optics in Astronomy. Cambridge University Press

Roddier N., 1990, Opt. Eng. 29(10), 1174

Rousset G., 1999, in (Roddier 1999), Chapt. 5, pp. 91-130

Rousset G., Fontanella J.-C., Kern P., 1990, A\&A 230, 29

Sasiela R.J., 1995, Electromagnetic Wave propagation in Turbulence Evaluation and Application of Mellin Transforms. Springer-Verlag

Thiébaut E., Conan J.-M., 1995, J. Opt. Soc. Am. A 12(3), 485

Véran J.-P., Rigaut F., Maître H., Rouan D., 1997, J. Opt. Soc. Am. A 14(11), 3057

Vernin J., Munoz-Tunón C., 1992, A\&A 257, 811

Voitsekhovich V.V., Bara S., 1999, A\&AS 137, 385

Véran J.-P., 1997, Ph.D. Thesis, Université de Paris XI 